Linguistique, littérature, didactique

\title{
L'interaction entre figuralité et registres discursifs
}

Interaction between Figurativity and Discursive Registers

\section{Marc Bonhomme}

\section{(2) OpenEdition \\ Journals}

Édition électronique

URL : http://journals.openedition.org/pratiques/2391

DOI : 10.4000/pratiques. 2391

ISSN : 2425-2042

Éditeur

Centre de recherche sur les médiations (CREM)

\section{Référence électronique}

Marc Bonhomme, "L'interaction entre figuralité et registres discursifs », Pratiques [En ligne], 165-166 | 2015, mis en ligne le 01 octobre 2015, consulté le 01 mai 2019. URL : http:// journals.openedition.org/pratiques/2391; DOI : 10.4000/pratiques.2391

Ce document a été généré automatiquement le 1 mai 2019.

(c) Tous droits réservés 


\title{
L'interaction entre figuralité et registres discursifs
}

\author{
Interaction between Figurativity and Discursive Registers
}

\author{
Marc Bonhomme
}

\section{Introduction}

1 Si les relations entre figures et genres ont retenu l'attention des théoriciens depuis des siècles ${ }^{1}$, on s'est beaucoup moins intéressé à l'interaction entre la figuralité et une autre famille de productions textuelles : celle des registres discursifs. Ceux-ci constituent certes une notion récente proposée il y a une quinzaine d'années par différents chercheurs ${ }^{2}$, laquelle réaménage les concepts de «types de discours » (Bronckart, 1996) et de "ton " (Soler, 2001), tout en s'opposant aux genres. Autant ces derniers (comme la comédie) définissent globalement des catégories métadiscursives historiquement et socioculturellement situées, autant les registres (comme le comique) désignent des patrons d'écriture fondamentaux et transhistoriques qui conditionnent la tonalité des discours.

2 Par ailleurs, la catégorie des registres est encore instable, posant au moins trois problèmes. Le premier est celui de leur dénombrement aléatoire suivant les théoriciens ${ }^{3}$. Le second problème concerne l'interférence des registres avec certains genres. C'est le cas pour l'épidictique et le délibératif traditionnellement envisagés comme des "genres oratoires » (Perelman \& Olbrechts-Tyteca, $1988: 692)$. Cependant, on peut distinguer les " genres » épidictique et délibératif, codifiés par la rhétorique de l'Antiquité gréco-latine, de patrons d'écriture - ou registres - traversant les époques et les cultures, qui sont épidictiques ou délibératifs et qui débordent les genres du même nom. Mais le problème le plus délicat des registres est celui de leur principe définitoire. Celui-ci est-il psychologique, consistant en «des attitudes affectives», comme le soutient A. Viala (2001: 171) ? Par exemple, le tragique serait corrélé à l'angoisse, le comique à la gaieté, le polémique à la colère ou le satirique à l'indignation. En fait, selon l'opinion de plusieurs linguistes (dont D. Maingueneau, 2008), le principe définitoire des registres est plutôt 
pragmatique, ceux-ci reposant sur des macro-actes qui régulent les productions verbales, tout en orientant leur interprétation. Ainsi, chaque registre est centré sur une tonalité illocutoire spécifique : faire rire de $x$ pour le comique, se moquer de $x$ pour le satirique, faire savoir $x$ pour le didactique, conseiller $x$ pour le délibératif, se plaindre de $x$ pour l'élégiaque, être ému par $x$ pour le pathétique, etc. De plus, les registres se développent autour de scripts indéfiniment actualisables dans les énoncés, avec une double articulation production/réception, à l'image du registre épidictique axé sur l'éloge et schématisable comme suit ${ }^{4}$ :

Figure 1. Script du registre épidictique

\begin{tabular}{|cc|}
\hline Énonciatcur Valoriser & Telle notion remarquable \\
PRODUCTION & RECEPTION discours élogieux \\
\hline
\end{tabular}

3 Sur ces bases, on peut se demander en quoi le domaine des figures entretient des liens privilégiés avec les registres. Dans un premier temps, nous verrons que celles-ci permettent à l'énonciateur de mettre en évidence la tonalité du ou des registres qu'il produit dans son discours. Nous nous interrogerons ensuite sur les motivations plus ou moins fortes qui existent entre figures et registres. Puis nous examinerons comment les figures sont elles-mêmes à la source d'effets-registres avant d'observer les relations plus larges entre figuralité, registres et genres.

\section{La situation des figures par rapport aux registres discursifs}

D’une façon générale, comment situer le champ des figures par rapport aux registres? Peut-on parler de figures du polémique, du didactique ou du délibératif? Plusieurs analystes n'ont pas manqué de relever des accointances entre telle figure et tel registre. B. Canal (1993) rattache la comparaison dépréciative au satirique, ou M. Blatter-Le Floch (2000) établit une liste des « choix lexicaux et rhétoriques » associés à l'épidictique, dans laquelle elle énumère des figures comme le paradoxe et la gradation. De son côté, J.M. Defays (1996) voit dans le comique la manifestation de figures primaires qui en soustendent les principales réalisations : le burlesque serait attaché à l'hyperbole à travers ses exagérations, l'humour à la litote du fait de ses condensations, ou encore l'absurde aux figures de l'inversion (chiasme, paradoxe).

Ces observations sont intéressantes, mais elles ne rendent pas suffisamment compte de l'articulation entre figuralité et registre. Pour l'élucider, considérons le registre polémique dont le script se présente ainsi : 
Figure 2. Script du registre polémique

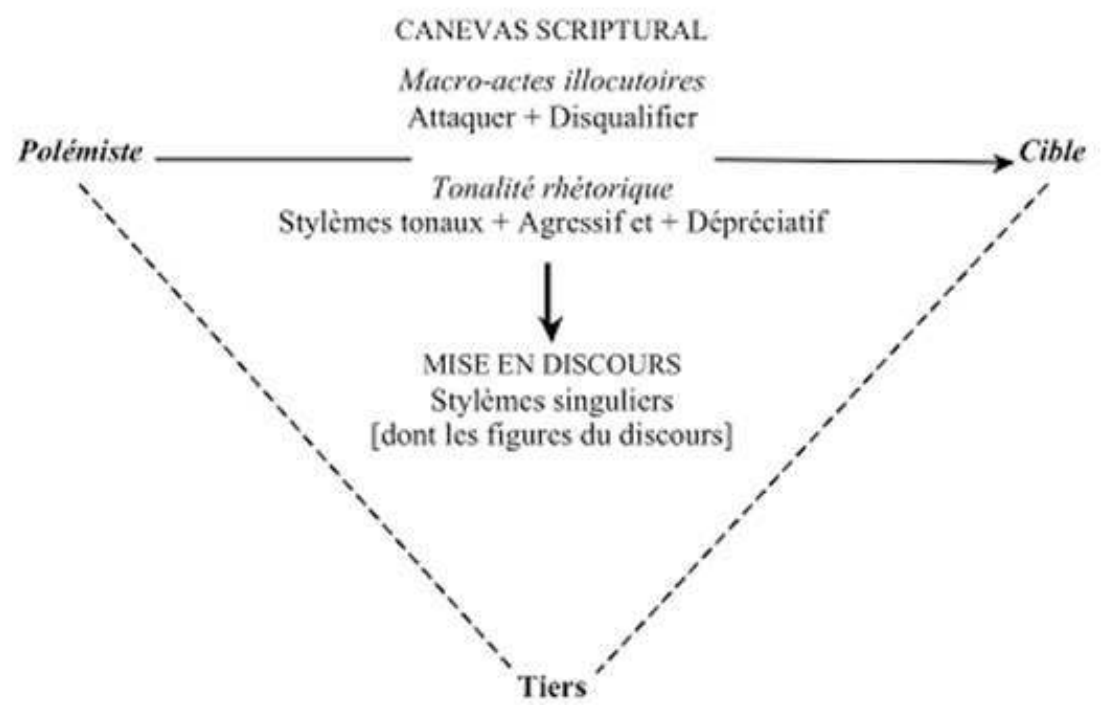

6 Ce script repose sur un canevas scriptural constitué de deux macro-actes illocutoires complémentaires, d'ordre actantiel (attaquer) et axiologique (disqualifier), entre un polémiste et sa cible vis-à-vis de tiers (témoins, lecteurs...) qu'il s'agit de persuader dans le sens du polémiste et de dissuader par rapport à la cible. Ce canevas scriptural comporte en outre une tonalité rhétorique qui oriente la mise en discours du registre polémique dans le cadre de ses macro-actes et que nous appréhendons en terme de stylèmes tonaux ${ }^{5}$ fondés sur les traits + Agressif et + Dépréciatif. Au niveau de son appropriation par le polémiste, un tel canevas scriptural peut être rendu par de nombreux procédés rhétoriques convergents - ou des stylèmes singuliers (Molinié, 1998) - qui contribuent à l'originalité de chaque discours polémique. Or, c'est parmi ces procédés que prennent précisément place les figures. Simplement, du fait de leur saillance et de leur densité informative, celles-ci donnent une portée accrue à la contextualisation du registre polémique.

7 Les figures apparaissent de la sorte comme des variations discursives surdéterminées qui mettent en avant la tonalité scripturale d'un registre, ce qu'illustrent deux textes polémiques. Le premier, À l'agité du bocal, constitue un pamphlet très connu de L.-F. Céline (2006 : 8-9) contre J.-P. Sartre :

«Il faut que je lise absolument, paraît-il, une sorte d'article, le Portrait d'un antisémite par J.-B. Sartre. [...] Oh! je ne veux aucun mal au petit J.-B. S. [...]. Mais page 462 , la petite fiente, il m'interloque ! Ah ! le damné pourri croupion! qu'ose-til écrire? "Si Céline a pu soutenir les thèses socialistes des nazis, c'est qu'il est payé". Textuel. Holà ! voici donc ce qu'écrivait ce petit bousier pendant que j'étais en prison. Satanée petite saloperie gavée de merde [...]! Anus Caïn pfoui. [...] Ici ! Que je t'écrabouille! Oui !... Je le vois en photo... ces gros yeux... ce crochet... cette ventouse baveuse... c'est un cestode! Que n'inventerait-il pas le monstre, pour qu'on m'assassine ! »

8 Dans ce pamphlet, les deux stylèmes tonaux du registre polémique sont explicitement actualisés par divers marquages rhétoriques, révélateurs de l'écriture célinienne. D'une part, le stylème + Agressif contre le territoire de J.-P. Sartre est concrétisé par des injures, des menaces, et surtout par deux figures saillantes: la figure morphologique de la siglaison déformante («J.-B. S. ») qui nie l'identité de la cible et la figure sémantique de la 
synecdoque particularisante qui réduit cette même cible à son fondement («la petite fiente », « anus »). D'autre part, le stylème + Dépréciatif contre l'image de J.-P. Sartre est rendu par une approximation péjorative («une sorte de») ou une minorisation disqualifiante ("petit»). Mais il trouve principalement son relief dans des métaphores négatives, de nature scatologique (« ce petit bousier »), démoniaque (« satanée », «Caïn ${ }^{6}$ ) ou tératologique (« le monstre »).

Le second texte est une épigramme de Voltaire contre É. Fréron (1983 : 109) :

« L'autre jour, au fond d'un vallon,

Un serpent mordit Jean Fréron :

Devinez ce qu'il arriva?

Ce fut le serpent qui creva. »

Dans cette épigramme, les stylèmes tonaux + Agressif et + Dépréciatif paraissent quelque peu estompés en raison de sa stylisation à travers les figures de l'ironie (mise en scène d'un mundus inversus) et de l'enthymème (implicitation de la conclusion ${ }^{7}$ ). Néanmoins, l'interprétation de cette épigramme révèle rétroactivement qu'elle prend bien place dans le registre polémique avec ses deux stylèmes : le stylème + Agressif, Voltaire insinuant en fin de compte que la méchanceté d'É. Fréron est supérieure à celle du serpent, et le stylème + Dépréciatif, suggéré par la contextualisation animalisante d'Éd. Fréron.

\section{La motivation entre les figures et les registres discursifs}

11 Si les figures permettent une meilleure textualisation d'un registre par un énonciateur suivant ses objectifs, se pose la question de leur motivation. Loin d'être due au hasard, la présence de telle ou telle figure dans un registre répond à des conditions qu'il importe d'analyser.

\subsection{Co-orientation entre profil figural et registres}

$12 \mathrm{Au}$ degré fort, certaines figures sont candidates à s'intégrer dans certains registres par le fait que leur profil offre une co-orientation tonale avec ceux-ci. Ce cas concerne essentiellement des figures ayant un spectre fonctionnel restreint, dont on peut voir trois exemples.

\subsubsection{Hyperbole et registre épidictique}

13 Plusieurs rhétoriciens, dont Hermogène (1997) ou O. Reboul (1991), ont relevé les rapports étroits entre l'hyperbole et le registre épidictique. Concrètement, il est facile de vérifier cette convergence, que l'on prenne un extrait d'un poème encomiastique de J. Du Bellay $(1558: 180)$ :

« Vostre rare vertu, qui n'a point de seconde,

Et vostre esprit ailé, qui voisine les cieulx,

Vous ont donné le lieu le plus prochain des Dieux,

Et la plus grand'faveur du plus grand Roy du monde.» (sonnet 168)

ou un passage de la dissertation d'A. de Rivarol (1991 : 72-73) célébrant la langue française au XVIII ${ }^{\mathrm{e}}$ siècle : 
« Le français, par un privilège unique, est seul resté fidèle à l'ordre direct, [...] et on a beau par les mouvements les plus variés et toutes les ressources du style, déguiser cet ordre, il faut toujours qu'il existe ; [...] la syntaxe française est incorruptible. C'est de là que résulte cette admirable clarté, base éternelle de notre langue. »

Ces deux textes mettent en œuvre une rhétorique de l'éloge étayée par de nombreuses hyperboles aux configurations variées ${ }^{8}$. On y remarque une nette co-orientation entre la tonalité illocutoire magnifiante de l'épidictique (louer), son canevas fondé sur l'amplification et le trait constitutif de l'hyperbole : l'intensification. Cela ne signifie pas que toute hyperbole est épidictique, ne serait-ce que parce que le registre épidictique en sélectionne la polarité positive, l'auxèse ascendante, plutôt que la polarité négative qu'est la tapinose'. Par ailleurs, la dimension axiologique, à savoir la valorisation - ou moins souvent la dévalorisation-, n'est pas vraiment inhérente à l'hyperbole. Mais cela n'empêche pas que cette dernière paraît assez naturellement adaptée au registre épidictique.

\subsubsection{Les figures pathémiques}

Dans la même perspective, d'autres figures ont une orientation pathétique et plus largement pathémique ${ }^{10}$, ce qui a été amplement constaté par les rhétoriciens. Entre autres, pour le Pseudo-Longin (1993), l'asyndète - par ses effets d'accélération - et l'anaphore - par son ressassement - mettent en branle les passions. Pour sa part, Hermogène (1997 : 366) répertorie des «figures de la véhémence ", parmi lesquelles l'apostrophe apparaît la plus symptomatique. De son côté, B. Lamy (1701) établit, par opposition aux tropes, une correspondance directe entre plusieurs figures et le psychisme de leur producteur. Ainsi, d'après lui, l'hyperbate, l'antithèse ou l'ellipse s'expliquent par différents troubles affectifs, dus au désordre ou à la rapidité des réactions émotives que le langage doit malgré tout exprimer. Quant à C. Batteux (1774: 128), il intègre dans ses «figures touchantes» des configurations comme l'exclamation ou l'imprécation qui marquent la fureur et le désespoir.

17 À partir de cette énumération décousue, il est possible de dégager quelques traits qui prédisposent les figures en question à actualiser le registre pathémique et à conférer au discours une valeur illocutoire de pathémisation. Parmi ces traits, on relève la tension discursive, nécessaire pour traduire les effets de maximalisation énergétique du pathos (cas de l'exclamation, de l'apostrophe ou de l'imprécation). On relève également la conflictualité sémantique, importante pour exprimer le choc des valeurs associé aux perturbations psychiques (cas de l'antithèse). On relève surtout la dislocation syntaxique, imputable aux effets de rupture produits par les affects dans la construction des énoncés (cas de l'hyperbate ou de l'ellipse). Tous ces traits apparaissent comme autant de stylèmes tonaux, virtuellement attachés à des figures spécifiques dont la fonction consiste, selon les cas, soit à révéler l'affectivité de leur énonciateur, soit à déclencher l'émotivité de leur énonciataire. Toutefois, si elles sont potentiellement pathémiques, ces figures n'ont un rendement pathémique effectif qu'à certaines conditions contextuelles.

\subsubsection{Figures et registre délibératif}

D'autres figures sont candidates, par leur profil même, à s'intégrer dans le registre délibératif, fondé sur le conseil avec ses phases de débat. Ces figures ont été mises en évidence par P. Fontanier (1977). Certaines d'entre elles sont prédisposées à actualiser le 
stylème tonal + Correctif dans une présentation modulée d'une opinion, à l'instar de l'épanorthose, «figure par laquelle on rétracte [...] ce qu'on vient de dire à dessein, pour y substituer quelque chose de plus fort, de plus tranchant ou de plus convenable» (ibid. 366). D'autres figures sont appropriées pour formuler le souhait sous-tendant le processus du conseil, à l'image de l'optation qui définit « l'expression d'un désir ardent d'obtenir pour soi ou d'autres quelque chose à quoi l'on attache [...] un grand prix » (ibid. : 438).

Mais les figures à orientation délibérative focalisent avant tout le stylème tonal + Manipulatoire lors de l'imposition d'un point de vue à travers un vernis dialogique. C'est le cas de l'interrogation oratoire qui vise à «indiquer la plus grande persuasion et [à] défier ceux à qui l'on parle de pouvoir nier» (ibid.: 368), de la subjection qui « subordonne [...] à une proposition, le plus souvent interrogative, une autre proposition le plus souvent positive, qui lui sert de réponse " (ibid. : 374 ), ou de la suspension qui réserve l'argument décisif pour la fin de l'intervention du locuteur afin de " produire une grande surprise ou une forte impression » (ibid. : 365).

\subsection{Congruence contextuelle entre figures et registres}

Au degré faible, l'intégration d'une figure dans un registre n'est plus motivée par le profil constitutif de celle-ci, mais le registre se borne à exploiter une structure disponible dans la figure en question, laquelle se trouve contextuellement en congruence avec sa tonalité illocutoire. Cette situation concerne les figures à large spectre fonctionnel, dont celles à pivot sémantique.

21 Soit la comparaison. Sa configuration A est comme B est suffisamment générique pour qu'elle s'intègre dans n'importe quel registre, notamment le registre comique quand celui-ci établit une similitude incongrue, à l'instar de la description de Quaresmeprenant dans le Quart Livre de Rabelais (1552), ou le registre lyrique lorsque le comparant met en lumière l'humeur plaintive de l'énonciateur, à l'exemple de J. Du Bellay (1967 : 74) en exil à Rome interpellant la France dans ses Regrets :

«Ores, comme un aigneau qui sa nourrice appelle,

Je remplis de ton nom les antres et les bois. » (sonnet 9)

Mais cette configuration A est comme B est aussi abondamment attestée dans la textualisation du registre didactique, en particulier dans le discours de vulgarisation des récits de voyage au $\mathrm{XVI}^{\mathrm{e}}$ siècle :

«Il se trouve davantage en Amérique une grande quantité de ces bêtes qu'ils nomment tapihire. [...] Ces bêtes sont de la grandeur d'un grand âne, mais le col est plus gros, et la tête comme celle d'un taureau d'un an. [...] Son poil est rougeâtre comme celui de certaines mules ou vaches de par-deçà » (Thévet, 1983 : 117).

ou dans les descriptions de monstres à la même époque :

« La mer Sarmatique [...] nourrit tant de poissons incogneus [...] et tant monstrueux que rien plus. Entre autres, il s'en trouve un tout ainsi fait qu'un limaçon, mais gros comme un tonneau, ayant les cornes quasi comme celles d'un Cerf. [...] Son nez est rondelet et faict comme celuy d'un chat » (Paré, 1971 : 113).

Dans ces cas, le registre didactique se limite à tirer parti d'une des nombreuses orientations sémantiques de la comparaison: celle où le comparant $B$ est davantage connu que le comparé $\mathrm{A}$. Cette orientation sémantique permet à l'énonciateur de transmettre un savoir nouveau ou difficilement intelligible, en établissant une proximité cognitive avec l'encyclopédie du récepteur. 
On pourrait faire des observations analogues sur les interactions entre les registres et les tropes comme la métaphore ou la métonymie. Du fait de la généricité de leur schème, l'analogie pour la première et la contigüité pour la seconde, elles prennent place dans les registres les plus variés. Grâce à la richesse de son imagerie, la métaphore peut revêtir tantôt une orientation épidictique, comme le montre la célébration de la femme dans «L'union libre » d'A. Breton (1966) :

«Ma femme à la chevelure de feu de bois

Aux pensers d'éclair de chaleur ".

tantôt une orientation didactique, comme l'illustre la reconceptualisation de la complexité de l'univers par l'analogie musicale chez H. Reeves (1981:200) :

"Quand je parle de «musique » ici, je m'exprime d'une façon analogique. C'est tout ce qui rend manifeste l'ordre somptueux de notre cosmos. [...] Devant ma fenêtre, une hirondelle plane, gracieuse. Elle apporte à manger à ses petits. [...] C'est, par analogie, la musique de la nature qui se manifeste ».

De même, la diversité des relations contigüelles de la métonymie la rend apte à donner des éclairages chaque fois pertinents aux registres qu'elle contextualise. Dans ce sens, la métonymie de la matière s'avère tout autant polémique dans le Dictionnaire philosophique de Voltaire ${ }^{11}$ que comique dans Zazie dans le métro de R. Queneau ${ }^{12}$.

\section{Figures et élaboration d'effets-registres}

Si grâce à leur rendement rhétorique les figures révèlent d'une façon saillante des registres qui leur sont préconstruits, elles participent aussi à l'élaboration d'effetsregistres dans la dynamique des discours. Cette action des figures sur les registres peut être abordée selon deux perspectives.

\subsection{L'action conditionnante des figures sur les registres discursifs}

En premier lieu, ce n'est jamais une figure isolée qui rend manifeste un registre, mais celui-ci émerge en contexte à travers la convergence de divers procédés figuraux et nonfiguraux. Cette genèse discursive d'un effet-registre est patente quand on examine un court extrait d'un violent texte d'A. Suarez (1915: 6-7), Nous et eux, composé en 1915, en pleine Première Guerre mondiale :

«L'Allemand est l'ennemi le plus fort et le plus atroce que peuple ait jamais eu. On a fait de lui l'automate de la destruction. Ses armées sont les légions infernales de la guerre. Il n'est plus homme que pour nuire et tuer. Sa méthode du mal est la perfection de toutes ses disciplines. [...] De leur dernier reptile, qui s'est nourri de notre pain, à leur dieu des armées coiffé de l'aigle, tous les mots qu'ils disent invoquent le massacre et la mort [...] : ce n'est que murs de fer et lacs de sang; le poing qui brise les crânes, la hache qui taille dans la chair vivante ».

Ce texte produit d'emblée un effet polémique qui se dégage d'un certain nombre de figures : synecdoque particularisante («L'Allemand»); hyperboles filées ( l'ennemi le plus fort et le plus atroce»...); paradoxe dépréciatif («Sa méthode du mal est la perfection »); redondance de métaphores à sémantique animalisante («reptile») et mécanisante («l'automate de la destruction »)... Nourri conjointement par ce cumul de figures, le registre polémique du texte émane en outre du lexique dysphorique utilisé (" tuer », « massacre ») et de sa composition répétitive en reformulations obsessionnelles. La polémicité de ce texte tient par ailleurs à ses conditions contextuelles, avec sa fonction 
persuasive de propagande antiallemande et sa fonction de renforcement de la cohésion nationale du public français.

Non seulement un registre se construit en contexte autour de figures caractéristiques, mais il arrive qu'une figure suscite contextuellement l'interprétation simultanée de deux registres. Soit cette réplique du capitaine Haddock extraite du Trésor de Rackham le rouge d'Hergé : «Oui, c'est votre faute, espèce d'analphabète diplômé ! ». Envisagé sous l'angle de la communication fictionnelle entre le capitaine Haddock et son interlocuteur Tournesol, l'oxymore "analphabète diplômé » constitue le point focal d'un registre polémique déployé dans le texte. D'une part, sa structure sémantique antinomique crée un clivage au sein du personnage de Tournesol, certes "diplômé » en sa qualité de professeur, mais surtout « analphabète » du fait de son incapacité à conduire l'expédition à l'endroit où se trouve le trésor de Rackham le Rouge. La teneur négative d'un tel oxymore est du reste renforcée par son insertion dans un cotexte d'injure interpellative (" espèce de!»). Mais, en même temps, si on le considère dans l'optique de la communication extrafictionnelle entre Hergé et ses lecteurs, l'énoncé «espèce d'analphabète diplômé !» active un registre comique, en raison de sa configuration burlesque et de sa thématique étrangère au domaine de l'injure. Il devient un exercice de style révélant la verve langagière d'Hergé, dans la lignée des nombreuses autres injures insolites prêtées au capitaine Haddock. On peut parler ici d'une syllepse ${ }^{13}$ tonale imbriquant deux registres l'un dans l'autre, ce qui contribue au charme des albums d'Hergé.

\subsection{Entre construction et déconstruction figurale des registres discursifs}

Une autre façon pour les figures de se réapproprier les registres à leur niveau est d'être au cœur de leur construction/déconstruction dans le déroulement d'un texte. Ce processus opère parfois à l'intérieur d'un même registre, comme le montre "Le pain", tiré du Parti-pris des choses de F. Ponge (1942: 46) :

\footnotetext{
« La surface du pain est merveilleuse [...] comme si l'on avait à sa disposition sous la main les Alpes, le Taurus ou la Cordillère des Andes.

Ainsi donc une masse amorphe en train d'éructer fut glissée pour nous dans le four stellaire, où durcissant elle s'est façonnée en vallées, crêtes, ondulations, crevasses... Et [...] ces dalles minces où la lumière avec application couche ses feux, - sans un regard pour la mollesse ignoble sous-jacente.

Ce lâche et froid sous-sol que l'on nomme la mie a son tissu pareil à celui des éponges : feuilles ou fleurs y sont comme des sœurs siamoises soudées par tous les coudes à la fois. Lorsque le pain rassit ces fleurs fanent. [...]

Mais brisons-la : car le pain doit être dans notre bouche moins objet de respect que de consommation. »
}

Les deux premiers paragraphes consistent en un éloge de la surface du pain, ce qui active le registre épidictique. Cet éloge s'effectue principalement par des figures analogiques, qu'elles soient comparatives ou métaphoriques. Dans le premier paragraphe, on découvre une comparaison hypothétique à orientation hyperbolique qui établit une ressemblance entre la croute et des montagnes représentant symboliquement trois continents: l'Europe avec les Alpes, l'Asie avec le Taurus et l'Amérique avec la Cordillère des Andes. Dans le deuxième paragraphe, l'analogie géographique se prolonge lors de la cuisson du pain (« elle s'est façonnée en vallées, crêtes »), tout en se doublant d'une valorisation 
cosmique grâce à la métaphore du feu («le four stellaire »). Mais dès la fin de ce paragraphe, l'éloge épidictique se déconstruit en son contraire, le blâme - également épidictique ( $c f$. note 4$)$, lorsque F. Ponge décrit l'intérieur du pain. On constate alors une dévalorisation de la mie, à travers une péjoration lexicale («la mollesse ignoble»), puis des comparaisons et des métaphores négatives, d'ordre architectural (« ce lâche et froid sous-sol»), animal ("pareil à celui des éponges»), humain ("comme des sœurs siamoises ») ou végétal («ces fleurs fanent »). Cette mise en discours de l'endroit et de l'envers du registre épidictique, entre éloge macrocosmique de la croute et blâme microcosmique de la mie, s'achève - comme souvent chez F. Ponge - par un brusque retour à la réalité : celle de la consommation alimentaire.

Dans d'autres contextes, le travail de déconstruction autour d'un pivot figural fait basculer le texte d'un registre à un autre, ce qu'on observe avec le Dictionnaire des idées reçues de G. Flaubert (2002 : 4-6) :

«Airain. Métal de l'antiquité.

Air. Toujours se méfier des courants d'air.

Alcoolisme. Cause de toutes les maladies modernes.

Ange. Fait bien en amour et en littérature.

Antiquités (Les). Sont toujours de fabrication récente. »

Ces extraits mettent en place le registre didactique, en accord avec le genre «dictionnaire» annoncé : entrées lexicales classées alphabétiquement, elles-mêmes suivies de définitions visant à éclairer le savoir du lecteur; structures elliptiques et sécheresse rédactionnelle de ces définitions... Cependant, si celle d'Airain semble conforme au registre décliné, plusieurs indices mettent en doute la visée informative des autres. On relève notamment une discordance entre l'entrée et la spécificité extrême de la définition consécutive (Air) ou une contradiction entre l'entrée et celle-ci (Antiquités). À cela s'ajoutent des hyperboles généralisantes («tous», «toujours»), la présence d'évaluatifs ("fait bien en amour ») ou une grande platitude dans la définition (Alcoolisme ). En réalité, comme le suggèrent les « idées reçues » du titre, il s'agit pour G. Flaubert de rapporter, dans un détachement critique dévoilé par ces indices, les poncifs de la pensée bourgeoise qu'il exècre. De la sorte, le registre didactique apparait comme une feinte, se dissolvant dans un registre satirique fondé sur la figure de l'ironie par mention (dans l'acception de D. Sperber \& D. Wilson, 1978).

\section{Figuralité, registres discursifs et genres}

Avec le Dictionnaire des idées reçues de G. Flaubert, on a entrevu l'influence contextualisante d'un autre facteur plus large dans l'interaction entre figures et registres : celui du genre. D'un côté, celui-ci peut exercer une fonction de filtre dans la figuralité des registres. Les genres comiques courts, telle l'histoire drôle, favorisent la mise en registre de figures ludiques sémantiquement concentrées, comme l'oxymore ou le paradoxisme, à l'image de cette histoire :

«-Papa, qu'est-ce qu'il y a sur cet arbre?

- C'est des prunes noires.

- Mais papa, elles ne sont pas noires, elles sont blanches.

- Oui. Elles sont blanches parce qu'elles sont vertes"

(Nègre, 1988 : 18).

Par contre, les genres comiques plus développés, telle la comédie théâtrale, permettent davantage un déploiement du registre comique sous la forme de figures répétitives, 
comme l'anaphore. En atteste la scène de L'Avare de Molière (I, 5) dans laquelle la réitération insistante de la formulation "sans dot» de la part d'Harpagon illustre le comique lié à la mécanisation du langage, théorisé par H. Bergson (1993).

Dans une perspective voisine, certains genres orientent la figuralité d'un registre. C'est le cas du genre « Avis de décès », textualisé dans cette annonce :

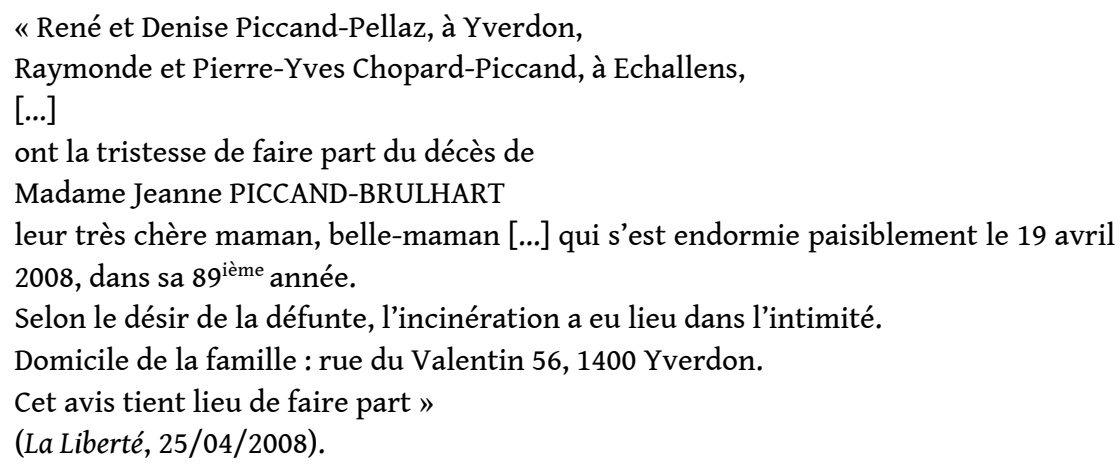

Ce texte repose certes sur le registre pathémique («tristesse »), mais avant tout sur le registre didactique avec un scénario très stéréotypé : faire savoir la mort de la personne concernée, puis les modalités de ses funérailles, ainsi que la composition et l'adresse de sa famille. Or, le contexte de décès qui conditionne le genre de ce texte suscite une euphémisation de son registre didactique, l'annonce de la mort se faisant par le biais d'une périphrase suggestive (« qui s'est endormie paisiblement »).

D'une façon plus problématique, il existe des brouillages non négligeables entre les marquages du genre et ceux du registre au sein des productions discursives. Si la situation est assez claire avec les genres mettant en œuvre des registres bien différenciés, ce qui est le cas du genre publicitaire ${ }^{14}$, il n'est pas facile de faire la part des choses entre figures du genre et figures du registre lorsque ces catégories entretiennent des relations étroites. Ainsi, dans le genre du pamphlet, à la fois pathémique et polémique, des figures comme la congerie ${ }^{15}$ ou l'oxymore recouvrent-elles des stylèmes tonaux, associés au registre polémique, ou des stylèmes génériques inhérents au pamphlet, comme le pense M. Angenot (1982) ? De même, dans une comédie, la mécanisation de l'anaphore est-elle un phénomène de registre, le comique, comme on l'a supposé précédemment avec L'Avare , ou de genre, la comédie? Elle participe sans doute aux deux, ce qui confirmerait la polyvalence fonctionnelle et catégorielle des figures du discours.

\section{Conclusion}

Nous avons réexaminé le statut des figures en les envisageant par rapport à l'une des catégories qui sous-tendent les productions verbales : celle des registres discursifs. Si les figures apparaissent comme un mode très efficace de leur actualisation et si certaines d'entre elles ont des affinités avec certains registres, elles contribuent à leur tour à construire et même à déconstruire des effets-registres à travers la contextualisation des textes et des discours. De surcroit, nous avons vu que cette interaction entre les registres et les figures implique aussi la catégorie des genres, ce qui complexifie le fonctionnement de ces dernières. Les quelques considérations que nous avons exposées sont des propositions à approfondir, dans la mesure où l'on est loin d'avoir exploré la problématique encore fluctuante des registres. Ainsi, nous avons analysé les liens entre la figuralité et les principaux registres, mais nous avons laissé dans l'ombre ses 
convergences possibles avec d'autres registres plus difficiles à cerner, comme le tragique, l'élégiaque ou l'épique. Quoi qu'il en soit, nous avons la confirmation que les figures sont de nature pragmatique, puisqu'elles tirent une partie de leur fonctionnalité des relations fructueuses qu'elles entretiennent avec leur cadre illocutoire et tonal constitué par les registres.

\section{BIBLIOGRAPHIE}

ADAM, J.-M. \& BONHOMme, M. (2012) [1997] : L'Argumentation publicitaire, Paris, A. Colin.

ANGENOT, M. (1982) : La Parole pamphlétaire, Paris, Payot.

Batteux, C. (1774) : Principes de la littérature, Paris, Saillant \& Desaint.

BERGSON, H. (1993) [1901] : Le Rire. Essai sur la signification du comique, Paris, Presses universitaires de France.

BlatTeR-Le Floch, M. (2000) : Le Blâme et l'éloge, Paris, Éd. Ellipses.

Bonhomme, M. (2005) : Pragmatique des figures du discours, Paris, H. Champion.

- (2008) : « De la pragmatique à la stylistique du registre épidictique », in : L. Gaudin-Bordes \&

G. Salvan (éds), Les Registres, Louvain-la-Neuve, Bruylant/Academia, p. 79-92.

BRETON, A. (1966) [1931] : «L'union libre » dans Clair de terre, Paris, NRF-Poésie/Gallimard.

BRONCKART, J.-P. (1996) : Activité langagière, textes et discours, Lausanne, Delachaux \& Niestlé.

CANAL, B. (1993) : «Quelques procédés satiriques dans Madame Bovary », Le Français dans tous ses états, 23, p. 46-50.

CÉLINE, L.-F. (2006) [1948] : À l'agité du bocal, Paris, Éd. L'Herne.

DEFAYS, J.-M. (1996) : Le Comique, Paris, Éd. Le Seuil.

DU BeLLAY, J. (1967) [1558] : Les Regrets, Paris, Gallimard.

FLAUBERT, G. (2002) : Dictionnaire des idées reçues, Paris, Éditions du Boucher.

FONTANIER, P. (1977) [1821, 1827], Les Figures du discours, Paris, Flammarion.

GAUDIN-BORDES, L. \& Salvan, G. (éds) (2008) : Les Registres, Louvain-la-Neuve, Bruylant/Academia.

HERGÉ (1945) : Le Trésor de Rackham le rouge, Bruxelles, Casterman.

HERMOGÈNE (1997) : L’Art rhétorique, Lausanne, Éd. L'Âge d'homme.

JAKOBSON, R. (1963) : Essais de linguistique générale, Paris, Éd. de Minuit.

JORDY, J. (2003) : La Mise en œuvre du programme de français en classe de seconde, rapport remis à M. le Ministre de l'Éducation nationale et de la recherche.

LAMY, B. (1701) : La Rhétorique ou l'art de parler, Paris, Mariette.

MAINGUENEAU, D. (2008) : « Les trois dimensions du polémique », in : L. Gaudin-Bordes \& G. Salvan (éds), Les Registres, Louvain-la-Neuve, Bruylant/Academia, p. 109-120. 
MOLIÈRE (1971) [1668] : L'Avare, Paris, Nouveaux Classiques Larousse.

MOLINIÉ, G. (1998) : Sémiostylistique, Paris, Presses universitaires de France.

NÈGRE, H. (1988) : Dictionnaire des histoires drôles, Paris, Fayard.

PARÉ, A. (1971) [1573] : Des monstres et prodiges, Genève, Droz.

PERELMAN, C. \& OlBRECHTS-TYTECA, L. (1988) : Traité de l'argumentation, Bruxelles, Éd. de l'Université de Bruxelles.

PONGE, F. (1942) : Le Parti pris des choses, Paris, Gallimard.

PSEUDO-LONGIN (1993) : Du sublime, Paris, Payot \& Rivages.

QUENEAU R. (1959) : Zazie dans le métro, Paris, Gallimard.

RABELAIS (1997) [1552] : Le Quart Livre, Paris, Éd. Le Seuil.

RAPIN, R. (1675) : Réflexions sur la poétique de ce temps, Paris, C. Barbin.

REBOUL, O. (1991) : Introduction à la rhétorique, Paris, Presses universitaires de France.

REEVEs, H. (1981) : Patience dans l'azur, Paris, Éd. Le Seuil.

RIVAROL, A. de (1991) [1783] : L'Universalité de la langue française, Paris, Arléa.

SOLER, P. (2001) : Genres, formes, tons, Paris, Presses universitaires de France.

SPERBER, D \& WILSON, D. (1978) : « Les ironies comme mention », Poétique, 36, p. 399-412.

SUAREZ, A. (1915) : Nous et eux, Paris, Émile-Paul Frères.

THÉVET, A. (1983) [1557] : Les Singularités de la France antarctique, Paris, Éd. La Découverte.

VIALA, A. (2001) : « Des “registres” ", Pratiques, 109-110, p. 165-177.

VOLTAIRE (1983) : «Épigramme contre Fréron », in : J.-M. Monod, La Férocité littéraire, Paris, Éd. La Table Ronde.

- (2008) [1784] : Dictionnaire philosophique, Paris, Classiques Garnier.

\section{NOTES}

1. Pensons notamment aux observations de R. Rapin (1675) et de C.Batteux (1774) sur la figuralité de la poésie lyrique ou, plus près de nous, aux considérations de R. Jakobson (1963) sur les rapports entre métonymie et récit réaliste.

2. Parmi lesquels A. Viala (2001) ou L. Gaudin-Bordes \& G. Salvan (2008). La notion de « registre discursif » ne doit pas être confondue avec les « registres sociolinguistiques » qui se rapportent aux variables d'une langue employées dans des situations sociales déterminées (registre soutenu, familier...).

3. Entre autres, A. Viala (2001) répertorie onze registres (le comique, le tragique, l'épique, le pathétique, le lyrique, l'élégiaque, le satirique, le polémique, le didactique, le délibératif, l'épidictique), quand J. Jordy (2003) n'en relève que sept (le comique, le tragique, l'épique, le pathétique, le lyrique, l'élégiaque, le fantastique).

4. Selon la tradition rhétorique, l'épidictique comporte aussi une polarité négative de dévalorisation orientée sur le blâme. Pour plus de précisions sur ce registre, voir M. Bonhomme (2008). 
5. Nous empruntons à G. Molinié (1998) la notion de stylème qui désigne toute unité de caractérisation stylistique.

6. Assassin de son frère Abel et premier homicide de l'humanité, Caïn a été condamné par Yahvé suite à son meurtre.

7. Cette épigramme renferme en effet seulement les deux premières phases de l'enthymème : la majeure (les trois premiers vers) et la mineure (le quatrième vers).

8. Les hyperboles s'y réalisent au moyen de superlatifs, de termes totalisants et de marqueurs d'exclusivité. Par la diversité de ses manifestations, l'hyperbole se range dans la famille des figures complexes (Bonhomme, 2005).

9. Celle-ci définit une hyperbole péjorative. Dans ce cas plus rare, l'éloge se transforme en blâme.

10. Dans la mesure où le terme pathétique est trop limité à certaines émotions fortes, comme la pitié, nous préférons le terme pathémique qui a l'avantage de recouvrir les manifestations plus englobantes de la passion.

11. Ainsi, quand Voltaire dénonce l'importation de la porcelaine chinoise en France: «Nous allons chercher à la Chine de la terre, comme si nous n'en avions point » (« Chine (de la)»).

12. Voir cette occurrence cocasse : «Charles s'avança jusqu'au zinc en bois depuis l'Occupation ». 13. La syllepse définit la coexistence de plusieurs sens ou de plusieurs niveaux de compréhension dans un même fragment discursif.

14. Comme l'ont montré J.-M. Adam et M. Bonhomme (2012), celui-ci combine les registres épidictique (éloge du produit) et délibératif (conseil d'achat).

15. Celle-ci forme une figure de construction qui accumule les composantes d'une situation dans un apparent désordre.

\section{RÉSUMÉS}

Cet article se propose d'étudier les relations entre les figures et les registres discursifs définis comme des matrices d'écriture qui conditionnent la tonalité des discours. Nous verrons d'abord comment, par leur saillance et leur densité informative, les figures donnent une portée accrue à la mise en texte des registres. Nous nous interrogerons par ailleurs sur les motivations qui incitent les figures à s'intégrer dans les registres. Si certaines d'entre elles offrent une forte affinité avec ces derniers (comme l'hyperbole avec le registre épidictique), d'autres figures - telle la métaphore - ne doivent leur présence dans les registres qu'à des facteurs contextuels. Nous examinerons ensuite comment les figures participent à la construction ou à la déconstruction d'effets-registres dans le déroulement des textes, avant d'analyser l'influence des genres dans l'interaction entre figures et registres. Au bout du compte, cet article confirme la nature pragmatique des figures, dans la mesure où leur fonctionnalité provient en partie des rapports qu'elles entretiennent avec leur cadre tonal et illocutoire constitué par les registres.

This paper studies the relations between figures and the discursive registers which are defined as matrixes of writing that determine discourse tonality. First, we will see how figures, through their visibility and their informative density, give a greater impact to the textual production of registers. Moreover, we will examine the motivations that incite figures to get integrated into registers. Some figures show a strong affinity with registers (such as hyperbole with the epidictic register), whereas others - such as metaphor - are present in registers only thanks to contextual factors. We will then analyze how figures contribute to the construction or to the deconstruction 
of effects-registers in the development of texts. Our paper will also study the influence of genres in the interaction between figures and registers. All things considered, we can confirm the pragmatic nature of figures: their functionality comes partially from the relations that exist between them and their tonal and illocutive frame, which is constructed by the registers.

INDEX

Mots-clés : contexte, figures, genres, registres, stylème, tonalité

Keywords : context, figures, genres, registers, styleme, tonality

\section{AUTEUR}

MARC BONHOMME

Université de Berne 